\section{Bericht über die Entwicklung eines AMDP-Moduls für Störungen durch psychotrope Substanzen}

Michael Lucht ${ }^{1}$, Sven Barnow ${ }^{1}$, Michael Krausz ${ }^{2}$, Wolfgang Maier ${ }^{3}$, Harald J. Freyberger ${ }^{1}$

${ }^{1}$ Klinik und Poliklinik für Psychiatrie und Psychotherapie der Ernst-Moritz-Arndt-Universität Greifswald im Klinikum Stralsund

2 Klinik und Poliklinik für Psychiatrie und Psychotherapie des Universitätsklinikums Hamburg-Eppendorf

${ }^{3}$ Klinik und Poliklinik für Psychiatrie und Psychotherapie der Universität Bonn
Die Arbeitsgemeinschaft für Methodik und Dokumentation in der Psychiatrie (AMDP) hat in den letzten Jahren erhebliche Anstrengungen unternommen, um neben dem klassischen AMDP-System [1] ergänzende Fremdbeurteilungsmodule zu verschiedenen Störungsgruppen zu entwickeln [2-4]. Ein Modul für die Beschreibung von Störungen durch psychotrope Substanzen fehlte bisher. Im folgenden Beitrag soll dargestellt werden, welche Leitlinien die Entwicklung dieses Suchtmoduls bestimmen werden. Als Kriterien zur Gewinnung des Merkmalsbestandes hat die AMDP die Folgenden festgelegt: Die Items sollen nach 1. Häufigkeit, 2. Spezifität, 3. Verlaufsempfindlichkeit, 4. Reliabilität und 5. klinischer Bedeutung konstruiert werden. Um über die bestehenden Klassifikationen hinaus eine Innovation in diesem Bereich zu ermöglichen, sollte es ein besonderes Ziel sein, vor allem der Verlaufsempfindlichkeit und klinischen Bedeutung entsprechende Beachtung zu schenken. Die Formulierung von AMDPItems im Bereich Störungen durch psychotrope Substanzen soll damit ein Handwerkszeug werden, dass für klinische und therapeutische Entscheidungen nutzbar ist. Hierzu wurde eine entsprechende Arbeitsgruppe gebildet.

In den neueren Klassifikationssystemen ICD-10 und DSM-IV wird der Bereich Störungen durch psychotrope Substanzen einerseits durch verschiedene Störungsbilder (Abhängigkeit, Missbrauch sowie Störungen, die Folgen von Substanzgebrauch beschreiben, wie kognitive Störungen oder Restzustände) abgebildet und andererseits für verschiedene Substanzen spezifiziert. Der Vielzahl der sich daraus ergebenden Kombinationen muss durch die Formulierung spezifischer Items Rechnung getragen werden. In dem hier vorliegenden Beitrag wollen wir am Beispiel der Alkoholabhängigkeit eine mögliche Strategie zur Formulierung von Items vorschlagen. Um eine klinische und Verlaufsvalidität der Items zu erreichen, ist es zunächst notwendig, ein ätiopathogenetisches Modell zu formulieren, dass die potenziellen Itembereiche in einen kausalen Zusammenhang stellt. Je nach Präferenz für ein bestimmtes biologisches, psychologisches oder soziologisches Modell sind verschiedene Ordnungsmöglichkeiten denkbar. Wir orientieren uns in unserem Modell am Ereignis „Rückfall“ bei Alkoholabhängigkeit. Der Rückfall bezeichnet gewissermaßen das Ergebnis des eine Abhängigkeitsstörung konstituierenden Symptoms, nämlich des Kontrollverlusts (ICD-10, DSM-IV). Eine Verminderung von Rückfällen ist

Suchttherapie 2001; 2: 25-27

(c) Georg Thieme Verlag Stuttgart · New York

ISSN 1439-9903 gleichzeitig das Ziel einer jeden Therapie der Alkoholabhängigkeit. Koob [5] formulierte, dass Abhängigkeit eine chronische, mit Rückfällen einhergehende Störung sei. Diese Störung sei charakterisiert durch den Drang zur Einnahme eines Suchtstoffs, der nicht kontrolliert werden könne. Von diesem Modell ausgehend haben wir in Abb. 1 Prädiktoren für Rückfälle dargestellt.

Die Abbildung beschreibt eine Vielzahl von Rückfallprädiktoren. Craving, der Drang nach einem Suchtstoff, gilt als Kardinalsymptom für den Rückfall. Psychotherapeutische (cue-exposure) und pharmakologische Strategien (Acamprosat, Naltrexon, Tiaprid) haben sich als rückfallvermindernde Modifikatoren dieses Parameters erwiesen [6-8]. Die Arbeitsgruppe von Verheul et al. [8] schlug drei unterschiedliche Craving-Subtypen vor („three-pathway-model“), die unterschiedlich empfunden werden (relief=Erleichterung oder reward $=$ Belohnung) und ätiologisch Dysfunktionen unterschiedlicher Neurotransmittersysteme zugeordnet werden können. Diesen Dysfunktionen könnten genetische Faktoren zugrunde liegen. Das Modell trägt damit der Heritabilität von Störungen durch Alkohol Rechnung [9].

Als weitere rückfallbestimmende Merkmale sind Temperamentsmerkmale bzw. Persönlichkeitszüge (novelty seeking) [10] sowie Depressivität und Ängstlichkeit als psychopathologische Variablen identifiziert [11,12] worden. Auch hier ist eine genetische Codeterminierung anzunehmen. Gerra et al. [13] fanden einen Zusammenhang zwischen positiver Familienanamnese und der Wirksamkeit des Antidepressivums Fluoxetin hinsichtlich der Rückfallprophylaxe bei Patienten mit Alkoholabhängigkeit. Einige Befunde sprechen für eine Assoziation von Temperamentsfaktoren mit Dopamin- und Serotoninrezeptorgenvarianten, wiewohl die Befundlage inkonsistent ist [14].

Des Weiteren werden Risiko, klinisches Bild und Verlauf der Alkoholabhängigkeit durch psychologische und soziale Faktoren mit determiniert. Obwohl eine Unterscheidung zwischen biologischen, psychologischen und soziologischen Bedingungen ein und desselben Gegenstandes arbiträr ist, folgen wir dieser Differenzierung aus Gründen der Verdeutlichung unserer Argumentation. Folgende neuropsychologische Modulatoren für Risiko und Verlauf von Alkoholabhängigkeit wurden untersucht: Alkoholwirkungserwartungen $[15,16]$, soziale Unterstützung, Motivation, Kognition [17] und Funktion des Arbeitsgedächtnisses (working memory) [18-20]. 


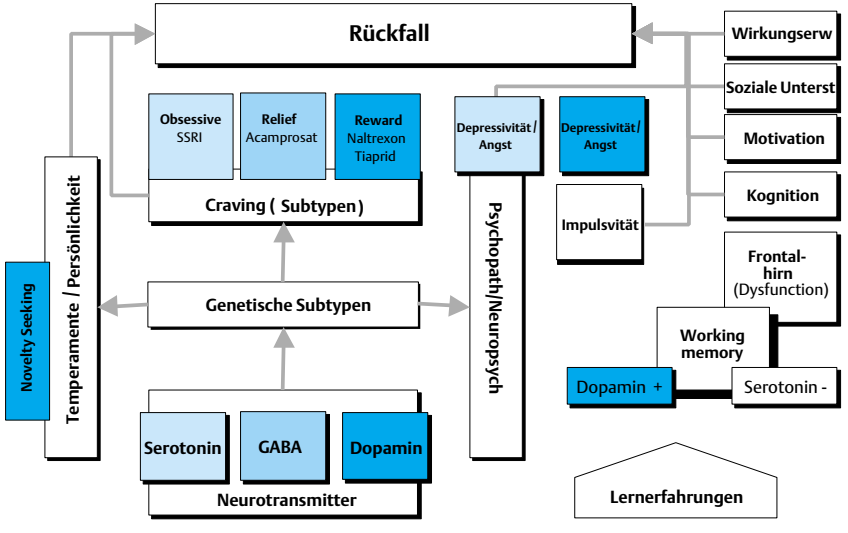

Abb.1 Vereinfachtes empirisches Modell für Biologie und Neuropsychologie des Rückfalls bei Patienten mit Alkoholabhängigkeit.

Das hier dargestellte Modell ist nicht in allen Punkten empirisch vollständig abgesichert, die Befunde beinhalten jedoch genügend konvergierende Evidenz, um eine Basis für das hier dargestellte Vorhaben der Formulierung eines empirisch abgesicherten und klinisch nutzbaren Moduls für Störungen durch psychotrope Substanzen zur Verfügung zu stellen. Das Modell ist uneingeschränkt offen für Ergänzungen und Modifikationen.

Wir wollen im Folgenden auf das Konstrukt Wirkungserwartungen als Paradigma für die Entwicklung eines Items für das Modul „Störungen durch psychotrope Substanzen“ eingehen: Wirkungserwartungen sind andauernde Überzeugungen über die Effekte von Alkohol (oder anderen Substanzen) [21]. Zahlreiche empirische Befunde belegen die Bedeutsamkeit von Wirkungserwartungen für den Verlauf von Störungen durch Alkohol: Motorische und kognitive Alkoholwirkungen werden unabhängig von der Art eines für den Probanden unbekannten Probetrunks (alkoholhaltiger vs. alkoholfreier Trunk) von den Wirkungserwartungen codeterminiert $[22,23]$, das Trinkverhalten von Adoleszenten wird von Wirkungserwartungen wesentlich mitbestimmt [16]. Rückfälle nach Behandlung von Alkoholabhängigkeit wurden durch Wirkungserwartungen prädiziert [24]. Eine genetische Mitbeteiligung zeigte sich darin, dass eine positive Familienanamnese mit positiven Wirkungserwartungen korreliert war. Des Weiteren wirkte der ALDH ${ }^{*}$ 2-Genotyp (steuert die Metabolisierung von Alkohol) protektiv gegen Alkoholkonsum via Verminderung positiver Wirkungserwartungen. Schließlich sind Alkoholwirkungserwartungen ein wichtiger Prädiktor für Selbstmedikation mit Alkohol bei Patienten mit Panikstörung. Wirkungserwartungen stehen in engem $\mathrm{Zu}$ sammenhang mit der erlebten Alkoholwirkung (level of response). Dieser level of response scheint eine genetische Codeterminierung aufzuweisen und bestimmt das Alkoholkonsumverhalten [25].

Dieses empirisch gut nachgewiesene Konstrukt wurde zudem psychopathometrisch gut untersucht, u.a. mit dem Alcohol Expectancies Questionnaire von Brown et al. $[15,26]$. Es wurden die folgenden Subskalen ermittelt: 1 . global positiv (+ ), 2. soziales und körperliches Wohlbefinden $(+)$, 3. soziale Ausdrucksfähigkeit (+), 4. Verstärkung sexuellen Erlebens (+), 5. Zunahme des Gefühls von Macht und Aggression (+),
6. Verminderung von Anspannung bzw, Entspannung (+), 7. kognitive und physische Einschränkung (-), 8. Gleichgültigkeit und Verantwortungslosigkeit (-). Die Subskalen standen im Zusammenhang mit hohem (+) oder niedrigem (-) Konsum von Alkohol. Unter 1260 zufällig aus der Normalbevölkerung ausgewählten Probanden zeigten sich eine Invarianz über Geschlecht und Ethnie sowie eine hohe Interkorrelation der Subskalen untereinander (Mittelwert $r=0,78 ; r=0,42$ bis $0,92)$. Die Autoren diskutierten eine bisher fehlende Spezifität bezüglich einzelner Subskalen in Hinblick auf spezifisches Verhalten. Umgekehrt ist jedoch davon auszugehen, dass die Subskalen austauschbare Verhaltensweisen mit uniformer Wirkung auf Trinkverhalten repräsentieren. Wir schlagen daher eine Zusammenfassung der Subskalen zu einem AMDPItem „Wirkungserwartungen“ vor:

Wirkungserwartungen (S; subjektives Item)

Definition: Der Patient erwartet das Auftreten von insgesamt als günstig und/oder angenehm empfundenen Konsequenzen durch den Konsum von Alkohol.

Erläuterungen und Beispiele: Die als angenehm empfundenen Konsequenzen umfassen beliebige Kombinationen der folgenden Bereiche: körperliches/seelisches Wohlbefinden; Erleichterung, soziale Kontakte zu schließen; eine Verbesserung sexuellen Erlebens; Erhöhung von Aggressivität und Durchsetzungsvermögen oder Verminderung von Spannung. Nachteile wie körperliche oder kognitive Einschränkungen oder Nachlässigkeit/Sorglosigkeit sind entweder nicht vorhanden oder fallen im Vergleich mit positiven Konsequenzen nicht ins Gewicht.

\section{Hinweise zur Graduierung:}

"leicht": Die Wirkungserwartungen sind nicht oder nur untergeordnet verhaltensbestimmend.

„schwer“: Die Wirkungserwartungen bestimmen Initiierung oder Rückfall in pathologisches Trinkverhalten wesentlich.

Wir gehen davon aus, dass dieses Item gestattet, im Sinne der oben beschriebenen Bedeutung der Wirkungserwartung, insbesondere den Verlauf bzw. Rückfall, zu prädizieren. Dies müsste in einer prospektiven Verlaufsstudie überprüft werden.

Die AMDP-Arbeitsgruppe hat zusammenfassend folgende Merkmalsbereiche identifiziert, aus denen heraus die betreffenden Items formuliert werden sollen:

1. Craving, 2. Konsummuster, 3. Konsumerwartungen/Funktionalität, 4. Erfahrungen mit Konsum, 5. Entzug allgemein und spezifisch und Komplikationen, 6. Neuropsychologie, 7. kategoriale Einordnung, 8. somatische Störungen, 9. Therapiebedarf (analog Addiction Severity Index), 10. Therapieerfahrungen, 11. subkulturelle Verankerung, 12. Legalsituation, 13. familiäre Belastung - soziale Situation, 14. Attributionsstile und Krankheitskonzept.

Es ist zu erwarten, dass spezifische Itemkonstellationen Patienten analog bekannter Alkoholtypologien kategorisieren. $\mathrm{Zu}$ nennen wären hier besonders die Typologie nach Clonin- 
ger (Typ I/II) sowie die Typologie nach Lesch (Typen I-IV) [27]. Beide Typologien sind besonders interessant, da sie Schnittmengen mit dem von uns zugrunde gelegten Modell aufweisen und ihrerseits Prognosen über Therapie und Verlauf der Alkoholabhängigkeit erlauben.

Die weiteren Arbeitsschritte der Arbeitsgruppe bestehen in a) der Formulierung von Items für die o.g. Merkmalsbereiche. Es muss dann b) eine Adaptation für verschiedene Substanzen erfolgen. Die Items werden c) durch Experten überprüft (Expertenvalidierung). Die Validierung des Itemkatalogs erfolgt d) in einer multizentrischen prospektiven Studie.

Die Arbeitsgruppe ist für Interessenten offen.

\section{Literatur}

${ }^{1}$ Das AMDP-System. In: AMDP (Hrsg). Manual zur Dokumentation psychiatrischer Befunde. Göttingen: Hofgrefe, 1995; 5. Auflage

${ }^{2}$ Grabe HJ, Hartschen V, Welter-Werner E, Thiel A, Freyberger HJ, Kathmann N, Boerner RJ, Hoff P. Entwicklung eines AMDPModuls zur Erfassung von Zwangssymptomen - Konzeptualisierung und empirische Ergebnisse. In: Maier W, Engel RR, Möller HJ (Hrsg). Methodik von Verlaufs- und Therapiestudien in Psychiatrie und Psychotherapie. Göttingen: Hogrefe, 2000: 214-217

${ }^{3}$ Das AMDP-System in der klinischen Anwendung und Forschung. In: Haug HJ, Stieglitz RD (Hrsg). Göttingen: Hogrefe, 1997

${ }^{4}$ Zwanzger P, Boerner RJ, Grabe HJ, Freyberger HJ, Möller HJ. AMDP-Modul zur Erfassung von Angststörungen. In: Maier W, Engel RR, Möller HJ (Hrsg). Methodik von Verlaufs- und Therapiestudien in Psychiatrie und Psychotherapie. Göttingen: Hogrefe, 2000: 212-213

${ }^{5}$ Koob GF. Neurobiology of addiction. Toward the development of new therapies. Ann NY Acad Sci 2000; 909: 170-185

${ }^{6}$ Shaw GK, Majumdar SK, Waller S, MacGarvie J, Dunn G. Tiapride in the long-term management of alcoholics of anxious or depressive temperament. Br J Psychiatry 1987; 150: 164-168

${ }^{7}$ Shaw GK, Waller S, Majumdar SK, Alberts JL, Latham CJ, Dunn G. Tiapride in the prevention of relapse in recently detoxified alcoholics. Br J Psychiatry 1994; 165: 515-523

${ }^{8}$ Verheul R, van den Brink W, Geerlings P. A three-pathway psychobiological model of craving for alcohol. Alcohol Alcohol 1999; 34: 197-222

${ }^{9}$ Schuckit MA. Genetics of the risk for alcoholism. Am J Addict 2000; 9: 103-112

${ }^{10}$ Meszaros K, Lenzinger E, Hornik K, Fureder T, Willinger U, Fischer G et al. The Tridimensional Personality Questionnaire as a predictor of relapse in detoxified alcohol dependents. The European Fluvoxamine in Alcoholism Study Group. Alcohol Clin Exp Res 1999; 23: 483-486

${ }^{11}$ Hodgins DC, el-Guebaly N, Armstrong S. Prospective and retrospective reports of mood states before relapse to substance use. J Consult Clin Psychol 1995; 63: 400-407

${ }^{12}$ Schaffer A, Naranjo CA. Recommended drug treatment strategies for the alcoholic patient. Drugs 1998; 56: 571-585

${ }^{13}$ Gerra G, Caccavari R, Delsignore R, Bocchi R, Fertonani G, Passeri M. Effects of fluoxetine and Ca-acetyl-homotaurinate on alcohol intake in familial and nonfamilial alcoholic patients. Current Therapeutic Research 1992; 52: 291-295

${ }^{14}$ Kuhn KU, Meyer K, Nothen MM, Gansicke M, Papassotiropoulos A, Maier W. Allelic variants of dopamine receptor D4 (DRD4) and serotonin receptor 5HT2c (HTR2c) and temperament factors: replication tests. Am J Med Genet 1999; 88: 168-172
${ }^{15}$ Brown SA, Christiansen BA, Goldman MS. The Alcohol Expectancy Questionnaire: an instrument for the assessment of adolescent and adult alcohol expectancies. J Stud Alcohol 1987; 48: 483-491

${ }^{16}$ Christiansen BA, Smith GT, Roehling PV, Goldman MS. Using alcohol expectancies to predict adolescent drinking behavior after one year. J Consult Clin Psychol 1989; 57: 93-99

${ }^{17}$ Noone M, Dua J, Markham R. Stress, cognitive factors, and coping resources as predictors of relapse in alcoholics. Addict Behav 1999; 24: 687-693

${ }^{18}$ Zhang XL, Begleiter H, Projesz B. Is working memory intact in alcoholics? An ERP study. Psychiatry Res 1997; 75: 75-89

${ }^{19}$ Finn PR, Justus A, Mazas C, Steinmetz JE. Working memory, executive processes and the effects of alcohol on Go/No - Go learning: testing a model of behavioral regulation and impulsivity. Psychopharmacology (Berl) 1999; 146: 465-472

${ }^{20}$ Sullivan EV, Rosenbloom MJ, Lim KO, Pfefferbaum A. Longitudinal changes in cognition, gait, and balance in abstinent and relapsed alcoholic men: relationships to changes in brain structure. Neuropsychology 2000; 14: 178-188

${ }^{21}$ MacAndrew C, Egerton RB. Drunken Compartment: A social explanation. Chicago: Aldine Publishing Co., Inc., 1969

${ }^{22}$ Marlatt GA, Rohsenow DJ. Cognitive processes in alcohol use: Expectancy and the balanced placebo design. In: Mello NK (Hrsg). Advances in Substance Abuse: Behavioral and Biological Research. Greenwich, Conn.: JAI Press, Inc., 1980: 159-199

${ }^{23}$ Hull JG, Bond CF. Social and behavioral consequences of alcohol consumption and expectancy: A metaanalysis. Psychol Bull 1986; 99: 347-360

${ }^{24}$ Brown SA, Goldman MS, Christiansen BA. Do alcohol expectancies mediate drinking patterns of adults? J Consult Clin Psychol 1985; 53: 512-519

25 Schuckit MA, Mazzanti C, Smith TL, Ahmed U, Radel M, Iwata N et al. Selective genotyping for the role of 5-HT2A, 5-HT2C, and GABA alpha 6 receptors and the serotonin transporter in the level of response to alcohol: a pilot study. Biol Psychiatry 1999; 45: 647-651

${ }^{26}$ George WH, Frone MR, Cooper ML, Russel M, Skinner JB, Windle $M$. A revised alcohol expectancy questionnaire: factor structure confirmation and invariance in a general population sample. J Stud Alcohol 1995; 56: 177-185

${ }^{27}$ Lesch OM, Walter H. Subtypes of alcoholism and their role in therapy. Alcohol Alcohol Suppl 1996; 1: 63-67

\section{Dr. med. Michael Lucht}

Klinik und Poliklinik für Psychiatrie und Psychotherapie der Ernst-Moritz-Arndt-Universität Greifswald im Klinikum Stralsund Rostocker Chaussee 70 18437 Stralsund

E-mail: lucht@mail.uni-greifswald.de 